\title{
Die sakramentale Bestimmung der Kunstfertigkeiten in den drei Prologen der, Schedula diversarum artium von Theophilus Presbyter
}

\author{
Gabriele K. Sprigath (München)
}

1961 hat Charles Reginald Dodwell in der Einführung zu seiner Ausgabe der ,Schedula' festgestellt: die gegenüber den drei Büchern mit den Rezepten nicht minder bedeutsamen drei Prologe seien oft der Aufmerksamkeit entgangen ${ }^{1}$. Das trifft bereits für die anfängliche Rezeption des Werkes zu, denn die zwischen dem 12. und dem 15. Jahrhundert entstandenen Abschriften der ,Schedula' enthalten zwar Rezepte aus den drei Büchern, die Prologe hingegen sind in ihnen nur vereinzelt zu finden ${ }^{2}$. Alle drei Prologe sind nur in den beiden ältesten, in der ersten Hälfte des 12. Jahrhunderts entstandenen, in Wolfenbüttel und Wien aufbewahrten Handschriften überliefert ${ }^{3}$. In der nach Lessings Wiederentdeckung der ,Schedula' einsetzenden Forschungsliteratur sind sie aus von vorgegebenen Deutungsmustern geprägten Blickwinkeln bisher stets selektiv gelesen und beurteilt worden ${ }^{4}$. Ich stelle deshalb zunächst in der hier gebotenen Kürze die wichtigsten

${ }^{1}$ Cf. Ch. R. Dodwell (ed.), Theophilus, De diversis artibus - The Various Arts, London 1961 [Neudruck Oxford 1986, 1998], x: ,[...] the prefaces, which are no less significant, have often escaped attention. “ J. Le Goff, Le travail dans les systèmes de valeur de l'occident médiéval, in: J. Hamesse/C. Muraille-Samaran (eds.), Le travail au Moyen Âge. Une approche interdisciplinaire. Actes du Colloque international de Louvain-la-Neuve, 21-23 mai 1987 (Publications de l'Institut d’Études médiévales: Textes, Études, Congrès 10), Louvain-la-Neuve 1990, 7 -21, hier 17: die ,Schedula' sei ,un texte d'une extrême importance non seulement par les renseignements qu'il donne sur les inventions et l'habileté technologiques, mais aussi par le contenu idéologique de ses prologues, trop peu souvent examinés mais extrêmement intéressants pour l'histoire médiévale“.

2 Cf. R. P. Johnson, The Manuscripts of the Schedula of Theophilus Presbyter, in: Speculum 13,1 (1938), 86-103.

3 Cf. Wolfenbüttel, Herzog August Bibliothek, Cod. Guelf. 69 Gud. lat. 2; Wien, Österreichische Nationalbibliothek, Cod. 2527.

4 Zum Forschungsstand cf. A. Speer/H. Westermann-Angerhausen, Ein Handbuch mittelalterlicher Kunst? Zu einer relecture der Schedula diversarum artium, in: C. Stiegemann/H. Westermann-Angerhausen (eds.), Schatzkunst am Aufgang der Romanik. Der Paderborner Dom-Tragaltar und sein Umkreis, München 2006, 249-258, hier 256: Die Autoren sprechen vom „Grundproblem der selektiven Lektüre und des Fehlens einer systematischen Analyse der gesamten Schrift sowie ihrer gattungs- und quellenmäßigen Einordnung". Exemplarisch dafür zuletzt H. Schlie, Ein „Kunststück“ Jan van Eycks in der Nachfolge der mittelalterlichen Artefakt- und Kunsttheorie, in: C. Laude/G. Heß (eds.), Konzepte von Produktivität im Wandel vom Mittelalter in die frühe Neuzeit, Berlin 2008, 243-286; M. H. Kowalewicz, Lessing et la culture du 
der in ihnen getroffenen Aussagen vor $(I)^{5}$. Anschließend gehe ich auf die Fragen nach dem „zugrundeliegenden Künstlerkonzept" und dem „mittelalterlichen Kunsterleben" ein (II) ${ }^{6}$.

\section{I.}

Das erste Wort der ,Schedula' und damit auch vom Prolog des ersten Buches ist der Name „Theophilus", mit dem der Verfasser sich als „,demütiger Priester, Knecht der Knechte Gottes, unwürdig des Namens und des Berufs Mönch“"vorstellt" ${ }^{7}$. Er wendet sich an die, „die die Untätigkeit des Geistes und das Umherschweifen der Seele durch nützliche Handbeschäftigung und ergötzliches Bedenken von Neuheiten meiden und verachten wollen, um der Erstattung himmlischen Lohnes willen" ${ }^{\star 8}$.

Auf die Einleitung folgt der Schöpfungsbericht: der ,nach dem Bilde Gottes und Gleichnis" geschaffene Mensch habe zwar „wegen der Schuld des Ungehorsams das Vorrecht der Unsterblichkeit verloren“, jedoch „die Würde von Wissen und Verständnis dem Geschlecht der Nachwelt bis hierher übertragen“. Wer "Sorgfalt und Mühe" hinzufüge, könne „die Befähigung zu aller Kunstfertigkeit und Begabung gleichsam als Erbrecht erlangen“" Die „menschliche Geschicklichkeit“ (sollertia bumana) sei zuerst auf „Gewinnsucht und Lüste“" (lucris et voluptatibus) ausgerichtet gewesen, dann aber mit der christlichen Religion zu ihrer von Gott vorhergesehenen Bestimmung, dem „Lob und Ruhm seines Namens" (ad laudem et gloriam nominis sui), gewendet worden ${ }^{10}$. Der Gläubige solle sich das ihm von Gott anvertraute „Erbe“ (hereditarium) aneigen und ohne Vorbehalte weitergeben. Theophilus stelle, was er „umsonst“ (gratis) dank der „Güte Gottes“ (benignitas Der) und seiner „Freigiebigkeit" (largitas) erhalten habe, in der ,Schedula' auch "umsonst" (gratis) zur Verfügung ${ }^{11}$.

Moyen Age (Spolia Berolinensia 23), Hildesheim 2003. Des Weiteren cf. B. Kurmann-Schwarz, "[...] quicquid discere, intelligere vel excogitare possis artium $[\ldots]$ “. Le traité De diversis artibus de Théophile, état de la recherche et questions, in: K. Boulanger/M. Hérold (eds.), Le vitrail et les traités du Moyen Âge à nos jours. Actes du XXIII ${ }^{e}$ colloque international du Corpus Vitrearum, Tours 3-7 juillet 2006, Bern-Berlin-Bruxelles-Frankfurt a. M.-New York-Oxford-Wien 2008, 29-44.

${ }^{5}$ Die Publikation der integralen Lektüre der Prologe und deren historische Kontextualisierung habe ich in Vorbereitung.

6 Speer/Westermann-Angerhausen, Handbuch (nt. 4), 255 und 257.

7 Theophilus, De diversis artibus, I, prol., ed. Dodwell (nt.1), 1: „Theopbilus, bumilis presbyter, servus servorum Dei, indignus nomine et professione monachi.“ Zur Übersetzung von servus mit „Knecht“ im Sinne von Leibeigener, cf. M. Parisse, Histoire et sémantique: de servus à homo, in: P. Freedman/M. Bourin (eds.), Forms of servitude in Northern and Central Europe. Decline, Resistance and Expansion (Medieval Texts and Cultures of Northern Europe 9), Turnhout 2005, 19-56.

8 Theophilus, De diversis artibus, I, prol., ed. Dodwell (nt. 1), 1; hier und im Folgenden eigene Übersetzung mit Kennzeichnung der Zitate durch Anführungszeichen.

${ }^{9}$ Ibid.

${ }^{10}$ Ibid., 1 sq.

11 Ibid., 2. 
Die ,Schedula“ sei ein „Schatz“ (thesaurus), aus dem der Leser - „Du, wer du auch bist, allergeliebtester Sohn" - ohne Anstrengungen und Kosten auswählen könne $^{12}$. Die Liste der Länder, aus denen Theopilus sein Wissen zusammengetragen habe, wird von „Griechenland“, sprich Byzanz angeführt ${ }^{13}$. Der Lernende soll die ,Schedula' durch häufiges Lesen im Gedächtnis (memoria) behalten und für Theophilus wegen seiner Uneigennützigkeit bei Gott im Gebet Barmherzigkeit erflehen. Die ,Schedula“ diene der „Ehre und dem Ruhm des Namens Gottes“ (bonor et gloria nominis eius) und dem „Fortschritt der Vielen" (profectus multorum) ${ }^{14}$.

Im Prolog des zweiten Buches ermahnt Theophilus den „allergeliebtesten Bruder“, den „Müßiggang“" aufzugeben und die „Nützlichkeit verschiedener Übungen ins Werk zu setzen“. Nichts lernen zu wollen sei „Schuld“ (culpa) [cf. Cato, Distichon, IV, 29, 2]. „Wissen" (scientia) hinzuzufügen sei „Arbeit" (labor) [cf. Sir. 1, 18], die zum „Fortschritt von Seele und Körper" (animae corporisque profectus) beitrage. Die Verfehlungen der Mönche im klösterlichen Leben würden Gott missfallen, dessen Augen auf dem Demütigen, dem Schweigsamen, dem im Namen Gottes Werkenden und auf demjenigen ruhen, der, der Vorschrift des Apostels Paulus [cf. Eph. 4, 28] folgend, um der Bedürftigkeit der Leidenden willen mit der Hand arbeite ${ }^{15}$.

Dessen „Nachahmer" (imitator) wünsche Theophilus zu werden ${ }^{16}$. Er habe sich der „heiligen Weisheit“ (agiae Sophiae) genähert und „erblicke die Kammer, die mit aller Mannigfaltigkeit unterschiedlicher Farben ausgestattet ist und die Nützlichkeit und Natur einer jeden zeigt ${ }^{c 117}$. Alles habe er ,in sorgfältiger Erfahrung im

12 Ibid., 3. Zur Form der Anrede cf. A. A. Häussling, Mönchskonvent und Eucharistiefeier. Eine Studie über die Messe in der abendländischen Klosterliturgie des frühen Mittelalters und zur Geschichte der Meßhäufigkeit (Liturgiewissenschaftliche Quellen und Forschungen 58), Münster 1973, 116 sq.: sie sei die Anrede des "geistlichen Vaters an den geistlichen Sohn“ auf Grund der „,bindenden Autorität“, der „Geistgebung des Vaters“ und ihrer „Herkunft aus der charismatischen Väterweisung“".

13 Theophilus, De diversis artibus, I, prol., ed. Dodwell (nt. 1), 4. Auf Griechenland (Byzanz) folgen: Russland, Arabien, Italien, Frankreich und Deutschland. Cf. J. Koder, Byzanz als Mythos und Erfahrung im Zeitalter Ottos I., in: B. Schneidmüller/S. Weinfurter (eds.), Ottonische Neuanfänge, Symposion zur Ausstellung „Otto der Grosse“, Magdeburg und Europa, Mainz 2001, 237-250, bes. 243-244: „Die Namen der Byzantiner.“

14 Theophilus, De diversis artibus, I, prol., ed. Dodwell (nt. 1), 4.

15 Op. cit., II, prol., 36.

${ }^{16}$ Cf. J. F. Hamburger, The medieval work of art: wherein the „work"? wherein the ,art"?, in: id./A.-M. Bouché (eds.), The Mind's Eye. Art and Theological Argument in the Middle Ages, Princeton 2006, 374 412, bes. 393: Der Rückbezug „dessen Nachahmer“ (huius imitator) zielt nicht auf Salomon, sondern auf Paulus.

17 Theophilus, De diversis artibus, II, prol., ed. Dodwell (nt. 1), 37 gibt „atrium agiae Sophiae“ wieder und vermerkt, worauf schon B. Bischoff aufmerksam gemacht hatte: Das Wort „atrium “ kommt in den beiden ältesten Abschriften in Wolfenbüttel und Wien nicht vor; cf. Quellengeschichtliche Untersuchungen zur ,Schedula Diversarum Artium' des Theophilus: Die Überlieferung des Theophilus-Rugerus nach den ältesten Handschriften, in: Münchner Jahrbuch der bildenden Kunst, 3. Folge, 3/4 (1952/53), 141-149, hier 148 [wiederabgedruckt in: id., Mittelalterliche Studien. Ausgewählte Aufsätze zur Schriftkunde und Literaturgeschichte, vol. 2. Stuttgart 1967, 174-182]. 
einzelnen erforscht, im Anschauen und mit den Händen klar genug erprobt". Als wissbegieriger Forscher habe er daran gearbeitet, die „Werknatur des Glases" (operam vitri naturam) zu verstehen und diese „Kunstfertigkeit“ (artificium) „durch Sehen sowie Hören “ erlernt ${ }^{18}$.

Der Prolog des dritten Buches ist in vier Gedankenschritten aufgebaut: (1) Der Dichter des Psalters König David als betender Büßer; (2) Auslegung der sieben Gaben des Heiligen Geistes auf die Tätigkeit des Kunstfertigen; (3) Die Wirkung der Bildwerke auf die Gläubigen; (4) Aufforderung an den Kunstfertigen, mit dem Herstellen der für den Gottesdienst benötigten Gerätschaften zu beginnen.

(1) Das erste Wort dieses Prologs ist der Name David. Gott habe ihn, ,seinem Herzen folgend“ [cf. Apg. 13, 22], als den „,vorzüglichsten der Propheten“ auserwählt und ihn, ,als Fürst über sein geliebtes Volk gesetzt und mit fürstlichem Geist gestärkt, damit er die Herrschaft eines so großen Namens edel und klug ordne“. David seinerseits habe, ,sich in der umfassenden Absicht seines Geistes in Liebe zu seinem Erbauer sammelnd, unter anderem dies gesagt: ,Herr ich habe den Schmuck deines Hauses geliebt ${ }^{6}[$ cf. Ps. 25, 8]“19. Dieses Haus habe er „Wohnung des himmlischen Hofes“ (babitatio coelestis) genannt, in dem „Gott über dem Hymnen singenden Engelschor in unermesslicher Klarheit throne“, und nach der er „mit allen Eingeweiden gelechzt" habe: „Eins habe ich vom Herrn erbeten, wonach ich suchen will, damit ich an allen Tagen meines Lebens im Hause Gottes wohne" [cf. Ps. 26, 4]. So bete „das Gefäß der frommen Brust und des allerreinsten Herzens, in dem Gott wirklich wohnt": „Erneuere den richtigen Geist in meinen Eingeweiden, Herr" [cf. Ps. 51,12]. Doch sei auch festzustellen, daß David auch „,den materiellen Schmuck des Gotteshauses als Ort des Gebetes begehrt habe“: oft habe er „allen Aufwand des Hauses des Herrn begehrt, im brennendsten Wunsch, selbst dessen Urheber zu werden“. Aber „wegen des wiederholten Vergießens von Menschenblut, wenn auch feindlichem", habe er es "nicht verdient" und Gold und Silber, Erz und Eisen seinem Sohn Salomon übergeben. Denn er habe im Buch Exodus gelesen [cf. Ex. 31, 1-11], „daß der Herr Moses den Auftrag gegeben hat, ein Tabernakel zu bauen und die Meister des Werkes namentlich auserwählt und sie mit dem Geist der Weisheit und des Verständnisses und des Wissens in allen Lehren erfüllt hat, um das Werk zu ersinnen" und ,,in Gold und Silber sowie Erz und Eisen, mit Edelsteinen und Holz mit aller Art von Kunstfertigkeit herzustellen“ ${ }^{\text {20 }}$. David habe ,in frommer Erwägung her-

18 Theophilus, De diversis artibus, II, prol., ed. Dodwell (nt. 1), 37.

19 Op. cit., III, prol., 61.

20 Den Namen Bezeleel (Ex. 31, 2) hat Theophilus nicht übernommen; zur Bedeutung von magister cf. M. Teeuwen, The Vocabulary of Intellectual Life in the Middle Ages (CIVICIMA. Études sur le vocabulaire intellectuel du Moyen Âge 10), Turnhout 2003, 95 sqq., bes. 95: „In the context of medieval intellectual life the term magister has a very broad meaning, in which two aspects are present: leadership and teaching. Magister can refer to someone who functions as a superior in a group of juniors or apprentices, to someone who has received a (university) education. [...]." 
ausgefunden“, daß „diese Art des Schmuckes“ Gott erfreue und ,angeordnet“, ihn ,,unter der Anleitung und Autorität des Heiligen Geistes“ anzufertigen ${ }^{21}$.

(2) Dazu befähige den Kunstfertigen ,die Gnade des siebengestaltigen Geistes“. 1. Durch den Geist der Weisheit (per spiritum sapientiae) ,erkennst du, daß alles Geschaffene von Gott ausgeht, und ohne ihn nichts ist". 2. Durch den Geist des Verstandes (per spiritum intellectus) „hast du die Fähigkeit der Begabung erhalten, auf welcher Ordnung, welcher Mannigfaltigkeit und welchem Maß du in deinen Werken bestehen sollst". 3. Durch den Geist des Rates (per spiritum consilii) „verbirgst du nicht dein dir von Gott zugestandenes Talent, sondern stellst es in Demut offen werkend und das Erkennen lehrend den Begehrenden getreu zur Verfügung“. 4. Durch den Geist der Stärke (per spiritum fortitudinis) „,vertreibst du alle Starrheit der Erschlaffung, und was du auch immer mit nicht langsamem Bemühen begonnen hast, führst du mit vollen Kräften zur Wirkung“. 5. Durch den Geist des „dir gewährten“ Wissens (per spiritum scientiae tibi concessum), „wirst du aus übervollem Herzen durch Begabung herrschen, und womit die Kühnheit des vollen Geistes dich vollständig erfüllt, wendest du öffentlich an“. 6. Durch den Geist der Frömmigkeit (per spiritum pietatis) „mäßigst du in frommer Erwägung, was, wem, wann, wieviel oder nach der Art des Werkes den Preis des Lohns [zu geben ist], so daß nicht das Laster des Geizes oder der Gier heranschleicht". 7. Durch den Geist der Furcht des Herrn (per spiritum timoris Domini) „beachtest $\mathrm{du}, \mathrm{da}$ du nichts aus dir selbst machen kannst, daß du nichts haben oder erdenken wollen kannst, was dir nicht von Gott zugestanden ist, sondern glaubend, vertrauend und mit Dank handelnd, schreibst du der göttlichen Barmherzigkeit $\mathrm{zu}$, was auch immer du weißt oder bist oder sein kannst ${ }^{\text {“22 }}$.

(3) Der Kunstfertige habe „das von ihm vertrauensvoll betretene Haus des Herrn mit so viel Anmut geschmückt" und den Betrachtern auf den Felderdecken (getäfelte Holzdecken) und den Wänden „das Bild des Paradieses Gottes in vielfältigsten Blumen frühlingshaft erblühend, in Gras und Blättern grünend, und die Seelen der Heiligen mit der Krone ihres verschiedenen Verdienstes erquickend“ gezeigt ${ }^{23}$. Damit habe er bewirkt, „daß sie Gott den Schöpfer in der Schöpfung loben und das Wunderbare in seinen Werken rühmen“. Denn nicht vermag das „menschliche Auge“ zu entscheiden, „auf welches Werk es zuerst den Blick heften soll: wenn es die Felderdecken betrachtet, erblühen sie wie Vorhänge; wenn es die Wände betrachtet, ist da das Bild des Paradieses; wenn es die Fülle des Lichtes aus den Fenstern erschaut, bewundert es die unschätzbare Zierde des Glases und die Mannigfaltigkeit des allerkostbarsten Werkes“24. Wenn die ,gläu-

21 Theophilus, De diversis artibus, III, prol., ed. Dodwell (nt. 1), 61 sq.

22 Ibid., 62 sq.

23 Cf. R. R. Grimm, Paradisus Coelestis Paradisus terrestris. Zur Auslegungsgeschichte des Paradieses im Abendland bis um 1200 (Medium Aevum 33), München 1977.

24 Zu dem Wort „Vorhänge“ cf. T. Klauser, Der Vorhang vor dem Thron Gottes, in: Jahrbuch für Antike und Christentum, Ergänzungsband 3 (1960), 141 sq. [Nachgedruckt in: id., Gesammelte Arbeiten zur Liturgiegeschichte, Kirchengeschichte und christlichen Archäologie, ed. von E. Dass- 
bige Seele das mit Linien so stark ausgedrückte Abbild des Leidens des Herrn erblickt", werde sie ,gestochen" (compungitur) ${ }^{25}$. "Wenn so viele Heilige in ihren Körpern die Qualen ertragen und so viele ewige Leben als Belohnungen erhalten werden, wird sie es gewahr und ergreift die Beachtung des besseren Lebens; wenn sie erschaut, wie viel Freude im Himmel und wie viele Marter der Flammen in der Hölle sind, wird sie durch die Hoffnung auf ihre guten Taten belebt und durch die Betrachtung ihrer Sünden und des Grauens erschüttert ${ }^{\star 26}$.

(4) Nun soll der „kluge Mann“ (vir prudens), „durch dessen Arbeit und eifriges Bemühen Gott so viele Brandopfer (holocausta) dargebracht werden“, die Gerätschaften herstellen, ,ohne die das göttliche Mysterium und der Dienst der Offizien nicht bestehen kann“: „Kelche, Leuchter, Rauchfässer, Messkannen, Krüge, Schreine für die heiligen Unterpfänder, Kreuze, Plenarien $[\ldots]^{\varsigma 27}$. Er beginne dies "mit Ordnung“" (ordine) $)^{28}$.

II.

Die drei Prologe sind in der Wolfenbütteler Handschrift dem jeweiligen Buch zugeordnet, in der Wiener Handschrift dagegen en bloc den drei Büchern vorangestellt ${ }^{29}$. In beiden Manuskripten sind sie nicht, wie in Dodwells Ausgabe, durch Absätze gegliedert, sondern fortlaufend geschrieben. Gegenüber den drei Büchern bilden sie einen eigenen, in sich abgeschlossenen Werkteil ${ }^{30}$. Beiden Werkteilen gemeinsam ist die Dreigliedrigkeit. Sie zeigt den Anspruch an, der die ,Schedula‘ von allen älteren Rezeptsammlungen unterscheidet: das Ausüben der in den

mann, Münster 1974, 218 sqq.]. Die Metapher „Vorhang“ ist in den Tabernakel-Kommentaren von Beda Venerabilis geläufig.

25 Theophilus, De diversis artibus, III, prol., ed. Dodwell (nt. 1), 63 sq. Zur compunctio (Zerknirschung, Reue) als der seit Gregor der Große gemalten Bildern zugesprochenen Wirkung cf. G. K. Sprigath, Zum Vergleich von scriptura und pictura in den Briefen von Papst Gregor d. Gr. an Serenus Bischof von Marseille, in: Jahrbuch für Internationale Germanistik, Jahrgang 41,2 (2009), 69-111, bes. 86 sqq.

26 Theophilus, De diversis artibus, III, prol., ed. Dodwell (nt. 1), 63 sq.

27 Cf. P. R. Schulte, Die Messe als Opfer der Kirche. Die Lehre frühmittelalterlicher Autoren über das eucharistische Opfer (Liturgiewissenschaftliche Quellen und Forschungen 35), Münster 1959.

28 Theophilus, De diversis artibus, III, prol., ed. Dodwell (nt. 1), 64. Zu ordo cf. die Forschungen zur historischen Metrologie in: D. Ahrens/R. C. A. Rottländer/F. Huber (eds.), Ordo und mensura, voll. I-VII (Siegener Abhandlungen zur Entwicklung der materiellen Kultur), St. Katharinen 1991-2002.

29 Cf. Bischoff, Untersuchungen (nt. 17), 146: „Ist der Gudianus seiner Erscheinung nach eine Bibliothekshandschrift, so die Wiener Handschrift mit ihrem ungewöhnlich kleinen Format von $126 \times 74$ mm eine zum persönlichen Gebrauch geschriebene Werkhandschrift."

${ }^{30}$ Cf. op. cit., 148: „[...] Die Prologe verleugnen nicht, daß sie nachträglich niedergeschrieben wurden; denn der Gedanke springt von dem einen zum nächsten über, als wenn die Rezepte gar nicht dazwischen ständen. So kann als sicher angenommen werden, daß sie im Konzept in einer Folge auf vorgehefteten Blättern standen und die Verteilung auf die Bücher für die Reinschrift vorgesehen war." 
drei Büchern verschrifteten handwerklichen Fertigkeiten und deren in den drei Prologen dargestellte sakramentale Bestimmung aufeinander zu beziehen.

Das erste Wort der ,Schedula“ ist der Name „Theophilus". Er bedeutet sowohl „der Gott Liebende“ wie „der von Gott Geliebte“. Die Doppelbedeutung kennzeichnet das Verhältnis von Gott und Mensch als auf gegenseitiger Liebe beruhend. Der Name geht auf die Legende zurück, nach der Theophilus von Adana in Kilikien einen Pakt mit dem Teufel eingeht, ihn später bereut und darauf von Maria errettet wird ${ }^{31}$. Der sich als Priester, Knecht der Knechte Gottes und Mönch ausweisende Verfasser der, Schedula' nimmt mit dem mittleren Titel eine Formel auf, die Papst Gregor der Große unter anderem auch in der an seinen Freund Leander von Sevilla gerichteten Anschrift zu seinem Werk, Moralia in Job“ mit Predigten zur Lebensführung der Mönche eingesetzt hat ${ }^{32}$. Sie steht für den apostolischen Auftrag zur Nachfolge Christi ${ }^{33}$.

Die Adressaten der ,Schedula“ seien diejenigen, „die die Untätigkeit des Geistes und das Umherschweifen der Seele durch nützliche Beschäftigung der Hand und das Bedenken ergötzlicher Neuheiten“ abwenden und bekämpfen wollen. Damit gibt sich die literarische Gattung zu erkennen, zu der die ,Schedula‘ gehört: der Priestermönch Theophilus hat sie in der monastischen Lebenswelt als Lehrschrift im Dienst des dem Mönchtum als gesellschaftlichem Stand zugewiesenen Bußauftrags verfaßt ${ }^{34}$. Er begründet ihn im Schöpfungsbericht (Prolog I) und stellt in

${ }^{31}$ Cf. G. G. Meersseman, Kritische glossen op de griekse Theophilus-legende (7 eeuw) en haar Latijnse vertaling ( 9 eeuw), in: Medelingen van de Koninklijke Vlaamse Academie voor Wetenschappen, Letteren en Schone Kunsten van Belgie, Klasse der Letteren, Jaargang XXV, Nr. 4 (1963), 1-36; K. Kunze/H. Linke, Theophilus, in: B. Wachinger (ed.), Deutsche Literatur des Mittelalters. Verfasserlexikon, vol. 9, Berlin 1995, 775-782, bes. 775; aus dem Griechischen von Paulus Diaconus (* ca. 720 ; † ca. 799), zusammen mit der Legende von Maria Aegyptica, ins Lateinische übersetzt und „Karl dem Kahlen gewidmet, wohl zur Förderung der Bußpraxis, denn in beiden Legenden ereilt die Gnade extreme, aber bußfertige Sünder. [...]“. Cf. A. Neumann, Teufelsbund und Teufelspakt (Mittelalter), in: G. Gersmann/K. Moeller/J.-M. Schmidt (eds.), Lexikon zur Geschichte der Hexenverfolgung, online: www.historicum.net/themen/ hexenforschung/lexikon/alphabethisch/p-z/art/Teufelsbund_und/html/artikel/5527/ca/ 1050b0c659/; zum Theophilusmirakel siehe „2.3. Die Teufelsbundliteratur des Mittelalters“.

32 Cf. Gregor der Große, Moralia in Job, ed. M. Adriaen (Corpus Christianorum. Series Latina 143), Turnhout 1979, 1; Dodwell (ed.), Theophilus (nt.1), XXXVI: die Formel sei vereinzelt bereits in Briefen unter anderem von Augustinus belegt und auch Bonifatius sowie Hrabanus Maurus bekannt gewesen. Ausführlicher Kommentar bei H. Fichtenau, Forschungen über Urkundenformeln, in: Mitteilungen des Instituts für österreichische Geschichtsforschung 94 (1986), 285-339, bes. 300 sqq. Cf. B. Reudenbach, Werkkünste und Künstlerkonzept in der Schedula des Theophilus, in: Stiegemann/Westermann-Angerhausen (eds.), Schatzkunst (nt. 4), 243-248, 245.

33 M. Fiedrowicz, Theologie der Kirchenväter. Grundlagen frühchristlicher Glaubensreflexion, Freiburg-Basel-Wien 2007, cf. 54-57: „Die Norm der Apostolizität.“

${ }^{34}$ Cf. P. Zerbi/C. D. Fonseca (eds.), Il monachesimo e la riforma eccleciastica (1049-1122), Atti della quarta Settimana internazionale di studio, Mendola 23-29 agosto 1968 (Miscellanea del centro di Studi medioevali 6) Milano 1971. Zum gesellschaften Stand der oratores neben dem der laboratores und dem der bellatores cf. O. G. Oexle, Die funktionale Dreiteilung der „Gesellschaft“ bei Adalbero von Laon. Deutungsschemata der sozialen Wirklichkeit im früheren Mittelalter, in: Frühmittelalterliche Studien 12 (1978), 1-54. 
dessen Dienst den mit der David-Figur legitimierten Schmuck des Gotteshauses (Prolog III).

Im Schöpfungsbericht ist der Mensch, da durch Einhauchen des göttlichen Odems beseelt und mit Vernunft begabt, als die Teilhabe an göttlicher Klugheit, Rat und Begabung verdienend vorgestellt ${ }^{35}$. Für Mönche geschrieben, kommt er ohne die in der Geschlechterpolarität begründete augustinische Erbsünden-Theologie aus ${ }^{36}$. Nicht in der Begierde (peccatum originale, concupiscentia) besteht die im Topos der „,teuflischen List“ extrapolierte Schuld (culpa) des Menschen, sondern allein in seinem Ungehorsam gegenüber Gott als Herrscher. Zwar werde der Mensch mit dem Verlust der paradiesischen Unsterblichkeit (exitus) bestraft, doch verbleibe ihm die Würde (dignitas) von Wissen und Verständnis (scientia, intelligentia).

Es ist die existentielle Furcht, der Liebe Gottes verlustig zu gehen, die Theophilus' Plädoyer für demütiges Lernen und für das Aneignen von Wissen durch das Lesen der ,Schedula' antreibt (Prolog I und II). Um den drohenden Verlust abzuwenden, soll der Mensch die von Gott umsonst erhaltenen Gaben auch umsonst weitergeben und in seinem Werk Gott zurückerstatten ${ }^{37}$. Der sakramentale Gabentausch ist das dritte Element in dem als auf Liebe und Gehorsam beruhend dargestellten Verhältnis von Gott und Mensch ${ }^{38}$. Er ist das Unterpfand für den Fortschritt (profectus) von Körper und Seele des Gläubigen auf seinem Weg der Rückkehr zu Gott (reditus) ${ }^{39}$. In der als von Gott ausgehend und mit den Werken des Menschen als zu Gott zurückkehrend beschriebenen Bewegung entwickele sich die menschliche Geschicklichkeit. Sie ist genealogisch strukturiert:

${ }^{35}$ Cf. T. Struwe, Die Wende des 11. Jahrhunderts. Symptome eines Epochenwandels im Spiegel der Geschichtsschreibung, in: Historisches Jahrbuch der Görres-Gesellschaft 112 (1992), 324-365, 337: der Autor zitiert einen Anfang der neunziger Jahre des 11. Jahrhunderts schreibenden Hersfelder Mönch: „Der Mensch wurde hiernach als ein mit Vernunft begabtes und mit dem Vermögen der Sprache ausgestattetes Wesen gekennzeichnet. Es verstand sich für den christlichen Autor von selbst, daß diese Vernunft nur als Teilhabe an der göttlichen Vernunft gedacht werden konnte.[...]"; dazu cf. ibid., nt. 48 sq.: Struwe sieht hier aristotelisches Gedankengut wirken.

${ }^{36}$ Cf. J. Gross, Geschichte des Erbsündendogmas. Ein Beitrag zur Geschichte des Problems vom Ursprung des Übels, 3 voll., München-Basel 1971.

37 Cf. G. Post/K. Giocarinis/R. Kay, The medieval heritage of a humanistic ideal: ,scientia donum dei est, unde vendi non potest', in: Traditio 11(1955), 195-234.

${ }^{38}$ Cf. M. Mauss, Essai sur le don. Forme et raison de l'échange dans les sociétés archaïques, in: L’Année Sociologique, nouvelle série, 1 (1923-1924), 30-186 [wiederabgedruckt in: id., Sociologie et anthropologie, Paris 1950, 143-279]; deutsche Übersetzung von E. Moldenhauer: Die Gabe. Form und Funktion des Austauschs in archaischen Gesellschaften, Frankfurt a. M. 1990; G. Weilandt, Geistliche und Kunst. Ein Beitrag zur Kultur der ottonisch-salischen Reichskirche und zur Veränderung künstlerischer Traditionen im späten 11. Jahrhundert (Archiv für Kulturgeschichte, Beihefte 35), Köln-Weimar-Wien 1992, 199-252: „Die zentrale Rolle des Königs“.

39 Cf. C. Heck, L'échelle céleste dans l'art du moyen âge. Une image de la quête du ciel, Paris 1997. W. Otten, From Paradise to Paradigm. A Study of Twelfth-Century Humanism (Brill's Studies in Intellectual History 127), Leiden-Boston 2004; E.-M. Kaufmann, Jakobs Traum und der Aufstieg des Menschen zu Gott. Das Thema Himmelsleiter in der bildenden Kunst des Mittelalters, Tübingen-Berlin 2006. 
die Gottesgaben werden im zweiten Glied an die Vorfahren und im dritten Glied als von Gott erhaltenes Erbe mit dem Erbrecht an die Leser der ,Schedula' weitergegeben.

Mit der David-Figur (Prolog III) überträgt Theophilus den sakramentalen Bußauftrag auf den Schmuck des Gotteshauses. In der mit dem Psalmvers 25, 8 („Herr, ich habe den Schmuck deines Hauses geliebt") ausgewiesenen Liebe Davids zu Gott legt er, der monastischen Tradition folgend, das Wort „Haus“ zuerst metaphorisch als Herz Davids und dann wörtlich als Gotteshaus aus, dem Ort des Gebets ${ }^{40}$. Mit den monastischen Tugenden Demut und Einfachheit gleicht er die David-Figur dem Mönchsideal an und stellt sie - im Unterschied zu den um 795 entstandenen ,Libri Carolini', in denen es lediglich heißt, der Tempel des Herrn sei ,,von David, dem allerheiligsten Priester, angeordnet und von Salomon dargebracht" (a David, sanctissimo vatum, dispositum et a Salomone dedicatum) - als königlichen Büßer dar ${ }^{41}$. Als solcher figuriert David neben Hiob auch in der um 817-822 von Hrabanus Maurus verfassten Schrift ,De institutione clericorum (II, 29: „De poenitentia“) und auf dem Krönungsbild der Bamberger Apokalypse (erste Hälfte des 11. Jahrhunderts) ${ }^{42}$. Demgegenüber setzt Theophilus einen eigenen Akzent, wenn er Davids kriegerisches Blutvergießen als Bußmotiv nennt. Es geht auf I Chronik 28, 3-7 zurück ${ }^{43}$. Doch während dort David seinem Volk mitteilt, Gott habe ihm den Bau des Tempels versagt, weil er ein Kriegsherr sei, ist es bei Theophilus der Schmuck des Gotteshauses, den David seinem Sohn Salomon überlassen muß.

Theophilus legitimiert ihn, indem er seine David-Figur als Leser des Buches Exodus vorstellt mit dem Bericht über den Moses von Gott erteilten Auftrag, das Tabernakel zu bauen und zu schmücken; Gott habe die „Meister des Werkes“ (magistros operum) mit dem Geist der Weisheit, des Verständnisses und des Wissens erfüllt $^{44}$. Dem fügt Theophilus zwei weitere Argumente hinzu: David habe herausgefunden, daß der Schmuck des Gotteshauses Gott erfreue, und er habe ange-

40 Weitere Beispiele für das Verknüpfen der beiden Auslegungen bei E. Dahl, Dilexi decorem domus Dei. Building to the glory of God in the Middle Ages, in: Acta ad Archaeologiam et Artium Historiam Pertinentia, Ser. alt. 1 (1981), 157-190.

${ }^{41}$ Cf. Theodulfus von Orléans, Opus Caroli regis contra Synodum (Libri Carolini), edd. A. Freeman/P. Meyvaert (Monumenta Germaniae Historica, Concilia 2, supplementum 1), Hannover 1998, 520 (1. 1-7).

42 Cf. Raban Maur, De institutione clericorum libri tres, II, 29, ed. D. Zimpel (Freiburger Beiträge zur mittelalterlichen Geschichte 7), Frankfurt a. M. 1996, 373-377. Cf. G. Suckale-Redlefsen/ B. Schemmel (eds.), Die Bamberger Apokalypse. Kommentar zur Faksimile-Ausgabe der Handschrift Msc. Bibl. 140 der Staatsbibliothek Bamberg, 2 voll., Luzern 2000, 69 sqq., und bes. 70 sq. die Beschreibung der Darstellung auf fol. 60r als „Der Triumph der Tugenden über die Laster“ mit Abbildung.

${ }^{43}$ Nachweis bei M. Kramp, Kirche, Kunst und Königsbild. Zum Zusammenhang von Politik und Kirchenbau im capetingischen Frankreich des 12. Jahrhunderts am Beispiel der drei Abteien Saint-Denis, Saint-Germain-des-Prés und Saint-Remi/Reims, Weimar 1995, 70, nt. 89.

${ }^{44}$ Cf. Exodus 31, 1-11. Dazu cf. A. G. Holder, The mosaic Tabernacle in Early Christian Exegesis, in: Studia patristica 25 (1993), 101-106. 
ordnet, daß er nur unter der Anleitung und mit der Autorität des Heiligen Geistes auszuführen sei. Auch der Psalter gilt als David vom Heiligen Geist eingegeben; in den Autorenbildnissen illustrierter Psalterhandschriften ist er als Taube dargestellt ${ }^{45}$. Der Schmuck des Gotteshauses ist damit in Gott selbst begründet und durch den Heiligen Geist sakralisiert.

Mit Hilfe dieser über die David-Figur vermittelten Argumentationskette überträgt Theophilus die seit frühchristlicher Zeit in der monastischen Lebenswelt geläufigen Auslegungen der sieben Gaben des Heiligen Geistes als die für den Aufstieg der Seele zu Gott erforderlichen ,Tugenden“ (virtutes, Prolog III) auf die Handwerker, die in der klösterlichen Lebenswelt den Schmuck des Gotteshauses herstellen ${ }^{46}$. Indem er sie auf deren Tätigkeiten auslegt, sakralisiert er diese und wertet sie damit gegenüber allen anderen Handwerken zu „Kunstfertigkeiten“ auf ${ }^{47}$. In den drei Büchern mit den Rezepten sind die von ihnen hergestellten Werke nach Materialien in zwei Gruppen unterteilt: die, die Farben und die, die feste Materialien verarbeiten.

Als mit Farben hergestellt nennt Theophilus drei Werkgruppen, die er, die Betrachter einbeziehend, mit affektiven Wirkungen besetzt (Prolog III): Wandmalereien und farbige Glasfenster ${ }^{48}$. Die vom Kunstfertigen aus festen Materialien durch „Arbeit und eifriges Bemühen“ (labor, studium) angefertigten Werke (Prolog III) seien „Brandopfer“ (bolocaust): es sind die von Theophilus aufgelisteten, für die Feier der Eucharistie erforderlichen Gerätschaften ${ }^{49}$. Ihre Qualität als „Brandopfer“ ergibt sich aus ihrer Unentbehrlichkeit für die Liturgie ${ }^{50}$.

Die beiden Materialgruppen liegen auch den drei Büchern mit den Rezepten zugrunde: Buch I und II enthalten diejenigen, die Farben, Buch III diejenigen, die feste Materialien verarbeiten. Dieses innere Ordnungssystem der drei Bücher

45 Cf. R. Kahsnitz, Der Werdener Psalter in Berlin, Ms. theol. lat. fol. 358. Eine Untersuchung zu Problemen mittelalterlicher Psalterillustrationen (Beiträge zu den Bau- und Kunstdenkmälern im Rheinland 24), Düsseldorf 1979, 168-189: „Das Autorenbildnis König Davids.“

46 Cf. Jesaias 11, 1-3. Dazu cf. W.-D. Hauschild, Pneumatologie in der Alten Kirche (Traditio Christiana 12), Bern 2004. Zur Auslegung der sieben Gaben des Heiligen Geistes von Augustinus über Gregor der Große bis zu Hrabanus Maurus cf. K. Boeckl, Die sieben Gaben des heiligen Geistes in ihrer Bedeutung für die Mystik nach der Theologie des 13. und 14. Jahrhunderts, Freiburg i. Br. 1931, 15-25; zu Gregor der Große cf. K. Ruh, Geschichte der abendländischen Mystik, vol. 1: Die Grundlegung durch die Kirchenväter und die Mönchstheologie des 12. Jahrhunderts, München 1990, 147-167; zum theologischen Umfeld cf. O. Lottin, La doctrine d'Anselme de Laon sur les dons du Saint-Esprit et son influence, in: Psychologie et morale aux XII et XIII siècles, vol. 4: Problèmes d’histoire littéraire de 1160 à 1300, Gembloux 1960, 445 - 477.

47 Ars habe ich mit „Kunstfertigkeit“ übersetzt, weil das Wort im Unterschied zu „Kunst“ und Kunstgewerbe" nicht mit Wertungen besetzt ist.

48 Theophilus, De diversis artibus, III, prol., ed. Dodwell (nt. 1), 63 sq.

49 Op. cit., 64. Cf. R. Huber/R. Rieth (eds.), Glossarium Artis. Deutsch-Französisches Wörterbuch zur Kunst, vol. 2: Das liturgische Gerät, Kreuze und Reliquiare der christlichen Kirchen/Les objets liturgiques, croix et reliquaires des églises chrétiennes, Tübingen-Strasbourg 1972.

50 Cf. E. Palazzo, Liturgie et société au Moyen Age, Paris 2000, bes. die Einleitung mit reichhaltiger Bibliographie, 17-39: „La messe dans la société médiévale." 
findet sich in dem um 1140 geschriebenen Katalog der verbrannten Bibliothek des Klosters von Sankt Blasius in Northeim bestätigt: dort ist unter der Nummer 72 der Titel ,Liber Theophili [non] de temperamentis colorum et opere fabrili` aufgeführt ${ }^{51}$.

Die Farben verarbeitenden Kunstfertigkeiten (Buch I und II) sind im Prolog des zweiten Buches auf die "agia sopbia“" ausgerichtet. Die griechische Formel begegnet in liturgischen Texten, wie zum Beispiel in der ,Missa de S. Sapientia von Alcuin ${ }^{52}$. Die gleiche Messe trägt im Leofric Missale den Titel ,Missa de sancta sapientia, que Christus est ${ }^{43}$. Das Gleichnis zwischen , sancta sapientia“ und Christus geht auf die patristische Exegese zurück: Christus als zweite Person der Trinität verkörpert den göttlichen Logos als die „,heilige Weisheit"“54. Die griechischen Worte „agia Sophia“ gelten als Spuren des seit dem 8. Jahrhundert in der Westkirche wirkenden ,griechischen“, sprich byzantinischen Einflusses ${ }^{55}$. Auch im Text des Theophilus ist „agia Sophia“ als liturgischer Bezug zu lesen, der die Farben verarbeitenden Kunstfertigkeiten sakralisiert, vergleichbar der Darstellung auf einer im letzten Viertel des 11. Jahrhunderts entstandenen Zeichnung zur „Psychomachie“ des Prudentius: Die in der Mitte des Kreises dargestellte weibliche Personifikation der Heilkunst ist nach oben auf die Christus ähnelnde Figur der „Heiligen Weisheit“" (sancta sapientia) über ihr ausgerichtet ${ }^{56}$.

In Buch I und II mit den Rezepten zu den Farben hebt Theophilus diejenigen Kunstfertigkeiten hervor, die Farben im Verbund mit Glas verarbeiten: ihnen ist ein ganzes Buch, das zweite der drei Bücher gewidmet, das mit dem Bau des Glasofens beginnt ${ }^{57}$. Es besteht aus 31 Kapiteln und ist damit fast genauso umfang-

51 Cf. H. Herbst, Mittelalterliche Bücherverzeichnisse des Benediktiner Klosters St. Blasius zu Northeim, in: Archiv für Kulturgeschichte 19 (1929), 346-368, 351.

52 Cf. Bischoff, Untersuchungen (nt. 17), 149, nt. 30.

53 Cf. F. E. Warren, The Leofric Missal as used in the Cathedral of Exeter during the Episcopate of its first Bishop, a.d. 1050-1972, Oxfordrough 1883, 176.

54 Cf. G. Florovsky, The Hagia Sophia Churches, in: Collected Works of Georges Florovsky, vol. 4: Aspects of Church History, Belmont, Mass., 1975, 131-135; M.-Th. d'Alverny, Le symbolisme de la Sagesse et le Christ de Saint Dunstan, in: Bodleian Library Record 5 (1956), 232-244.

55 Cf. Warren, Leofric (nt. 53), 176, nt. 2: „This is an instance of the occasional survival of Greek words in Western Liturgies“; M.-Th. d'Alverny, La sagesse et ses sept filles. Recherches sur les allégories de la philosophie et des arts libéraux du $\mathrm{IX}^{\mathrm{e}}$ au XII ${ }^{\mathrm{e}}$ siècle, in: Mélanges dédiés à la mémoire de Félix Grat, vol. 1, Paris 1946, 245-278, 257, nt. 1: „L'influence grecque est visible dans le culte rendu à la Sagesse en Occident à partir du VIII ${ }^{\mathrm{e}}$ siècle. [...].“

56 Cf. Lyon, Bibliothèque Municipale, Ms. Palais des Arts 22; Beschreibung bei R. Stettiner, Die illustrierten Prudentiushandschriften, Berlin 1895, 58 sq., Tf. 109,1. Zum Zusammenhang von , sapientia“ und Christus cf. auch M. Gibson, A picture of „Sapientia“ from S. Sulpice, Bourges, in: Transactions of the Cambridge Bibliographical Society 6 (1973), 126 sqq. [wiederabgedruckt in: ead., ,Artes' and Bible in the Medieval West (Variorum. Collected Studies Series 399), Aldershot 1993, 126-128].

57 Nach der Kapitelzählung von Theophilus, De diversis artibus, ed. Dodwell (nt. 1). Cf. F. Dell'Acqua, Glassmakers in the West between Late Antiquity and the Middle Ages, in: M. Beretta (ed.), When glass matters. Studies in the History of Science and Art from Graeco-Roman Antiquity to Early Modern Era (Bibliotheca di Nuncius 53), Firenze 2004, 135-150, zu Glasöfen 139-145, 
reich wie Buch I mit 38 Kapiteln zu den anderen, mit Farben arbeitenden Kunstfertigkeiten.

Den prominenten Platz dieser aus Glas hergestellten Werkgruppe in der ,Schedula' bestätigen drei Aussagen in den drei Prologen. Im Prolog des ersten Buches ist Frankreich wegen der dort geschätzten , kostbaren Vielfalt der Fenster“, wegen der Glasmalerei also, in der Länderliste aufgeführt. Im Prolog des zweiten Buches fasst Theophilus die in Buch I zusammengestellten Rezepte als „diese Art von Malerei, die nicht durchsichtig sein kann“ zusammen, um sie von den Kunstfertigkeiten abzugrenzen, die Farben und Glas im Verbund verarbeiten. Während er sonst von , ars“" spricht, hebt er an dieser Stelle im lateinischen Text diese Tätigkeiten ausdrücklich mit dem Wort „, artificium “ hervor. Im Prolog des dritten Buches ist von den Glasfenstern die Rede, durch die das „menschliche Auge“ die „Fülle des Lichts“ erschaue sowie vom „unschätzbaren Schmuck des Glases“ und der „Mannigfaltigkeit des allerkostbarsten Werkes “58. Offensichtlich galt das besondere Interesse des Theophilus den Verfahrensweisen, mit denen farbiges Glas hergestellt werden konnte. In deren schriftlicher Überlieferung nimmt die ,Schedula' denn auch einen zentralen Platz ein ${ }^{59}$.

In den drei Prologen entwirft der mit dem Mönchsnamen Theophilus ausgewiesene Verfasser der Lehrschrift ein Idealbild vom Kunstfertigen. Ihm liegen die Figuren des legendären Mönchs Theophilus (Prolog I) und der als Dichter des Psalters, als Prophet und als Büßer präsentierte König David (Prolog III) zugrunde. Sie verweisen auf das Verhältnis von Mönchtum und sakralem Königtum als gesellschaftliches Bezugsfeld ${ }^{60}$. Im mittleren Prolog ist der Liste mit den Ver-

bes. 144: „The only complete description that we have of medieval glass furnaces is that of Theophilus (lib. II, cap. II-III) who tells how to build a furnace for modelling the glass objects, and a second furnace for tempering them. [...]." Deutsche Übersetzung von E. Brepohl, Theophilus Presbyter und das mittelalterliche Kunsthandwerk. Gesamtausgabe der Schrift, De diversis artibus` in zwei Bänden, vol. 1: Malerei und Glas, Köln-Weimar-Wien 1999, 145 sq.

58

Theophilus, De diversis artibus, III, prol., ed. Dodwell (nt. 1), 63. Cf. F. Dell'Aqua, Lux et vitrum: the evolution of stained glass from the late roman empire to the gothic age, in: Beretta (ed.), glass (nt. 57), 221-248.

59 Cf. F. Dell'Acqua, La presenza/assenza dei vetrai nelle fonti scritte (secoli IV-XI), in: ead./ R. Silva (eds.), Il colore nel medioevo. Arte, simbolo, tecnica. La vetrata in occidente dal IV all' XI secolo, Atti delle Giornate di Studi, Lucca 23-24-25 septembre 1999 (Collana di studi sul colore 3), Lucca 2001, 195-211; K. Boulanger, Les traités médiévaux de peinture sur verre, in: Bibliothèque des Chartes 162 (2004), 9-33, bes. 13-17: die Autorin führt Theophilus als ältestes der von ihr behandelten fünf Dokumente auf.

${ }^{60}$ Cf. M. Bloch, Die wundertätigen Könige, München 1998; zur Tradition, Könige, Bischöfe und Priester durch die Salbung mit geweihtem Öl zu heiligen, 102-104, bes. 103: „Es lag nahe, die alten israelitischen Bräuche zur Gänze zu übernehmen und von der Salbung der Katechumenen oder Priester zur Salbung der Könige überzugehen. Das Beispiel Davids und Salomons erlaubte es, den Königen ihren geheiligten Charakter in einem christlichen Sinn wiederzugeben. " Cf. J. Wollasch, Kaiser und Könige als Brüder der Mönche. Zum Herrscherbild in liturgischen Handschriften des 9.-11. Jahrhunderts, in: Deutsches Archiv für die Erforschung des Mittelalters 40 (1984), 1-20. Zum sakralen Königtum cf. E. H. Kantorowicz, Die zwei Körper des Königs. Eine Studie zur politischen Theologie des Mittelalters, München 1990, Kap. 3, 64-104: „Königtum 
fehlungen und Verstößen der Mönche gegen die Regeln des klösterlichen Lebens die Trias der Zitate von Cato, Salomon und Paulus (Prolog II) gegenübergestellt: nicht lernen zu wollen sei, wie der Ungehorsam gegen Gott, „Schuld“ (culpa), und "Arbeit" (labor) sei es, das in der ,Schedula“ vorgefundene und durch die Tätigkeit des Lesens anzueignende „Wissen“ (scientia) zur handwerklichen Tätigkeit hinzuzufügen ${ }^{61}$.

Diese Bestimmung von „Arbeit“ (Prolog II) steht, zusammen mit der hohen Wertschätzung der Farben im Verbund mit Glas verarbeitenden Werkgruppe (Prolog II), im Mittelpunkt des aus den drei Prologen aufgebauten Textkörpers und damit im Zentrum der ,Schedula'. Der in der monastischen Lebenswelt sein Werk demütig herstellende Kunstfertige soll mit „ratio “ und ,intelligentia“ das in ihr versammelte „Wissen“ (scientia) seinem Gedächtnis durch wiederholtes Lesen einprägen und es im Dienst der sakramentalen Bestimmung seines Werkes seiner Tätigkeit hinzufügen ${ }^{62}$. Der Anspruch gilt ebenso für die ,Schedula' selbst: auch sie dient, wie die Werke der Kunstfertigen, dem Ruhm des Namens Gottes und dem Fortschritt (profectus) der Gläubigen auf ihrem Weg der Rückkehr zu Gott.

Als der Priestermönch Theophilus sich daran machte, sein derart konzipiertes Werk zu verfassen, hatte er unter den Lehrschriften keine Vorbilder zur Hand. Könnte er sich mit seiner Wahl der Dreigliedrigkeit für die Zuordnung von drei Büchern und drei Prologen im Aufbau der ,Schedula' an in der monastischen Lebenswelt einflußreichen Werken wie der aus drei Büchern bestehenden Schrift ,Historia ecclesiastica“ von Cassiodor oder naheliegender an der Schrift ,De institutione clericorum libri tres' von Hrabanus Maurus orientiert haben? Könnte er aber nicht auch aus den im klösterlichen Schulbetrieb vermittelten AristotelesFlorilegien das aristotelische Entwicklungsmodell von "Anfang-Mitte-Ende“ gekannt haben ${ }^{63}$ ?

und Christus.“ A. Grabois, Un mythe fondamental dans l'histoire de France au moyen âge: Le ,roi David“ précurseur du ,roi très chrétien', in: Revue historique 287 (1992), 11-31. F.-R. Erkens, Der pia Dei ordinatione rex und die Krise sakral legitimierter Königsherrschaft in spätsalischfrühstaufischer Zeit, in: J. Jarnut/M. Wernhoff (eds.), Vom Umbruch zur Erneuerung? Das 11. und 12. Jahrhundert: Positionen der Forschung (Mittelalterstudien des Instituts zur interdisziplinären Erforschung des Mittelalters und seines Nachwirkens 13), München 2006, 71-101.

${ }^{61}$ Cf. G. Keel, Laborare und Operari. Verwendungs- und Bedeutungsgeschichte zweier Verben für „arbeiten" im Lateinischen und Galloromanischen, Bern-St.Gallen 1942. Hamesse/Samaran (eds.), travail (nt. 1).

${ }^{62}$ Cf. M. Carruthers/J.M. Ziolkowski (eds.), The medieval craft of memory an anthology of texts and pictures, Philadelphia 2002.

${ }^{63}$ Cf. S. Herzberg, archê, in: O. Höffe (ed.), Aristoteles-Lexikon (Kröners Taschenausgabe 459), Stuttgart 2005, 68-76; Ph. Brüllmann/K. Fischer, meson, in: op. cit., 344 sqq.; F. Ricken, telos, in: op. cit., 575-583; W. Beierwaltes, Proklos. Grundzüge seiner Metaphysik (Philosophische Abhandlungen 24), Frankfurt a.M. 1965, Kap. I, 24-164: „Trias“, bes. 24-31: „1. Das Wesen von Trias als dialektische Einheit“, 24: „[...] die, triadische Gestalt' ist nicht ein dem Sein oder dem Denken äußerliches Klassifikations-,Schema', das nur formelle Bedeutung hätte, sondern konstitutives Element der Denkbewegung und jedwedes Seienden, das in je anderer Dimension von Seiendem je anders als immer mit sich selbig seiende Sinnstruktur erscheint." 
Dies sind zwei von vielen Fragen, die die Lektüre der, Schedula‘ aufwirft. Dazu kommt die in jüngster Zeit entstandene neue Forschungssituation: Albert Ilgs 1874 vorgeschlagene und seither nahezu als Gewissheit gehandhabte These, der Priestermönch Theophilus und der Goldschmied Roger von Helmarshausen seien identisch, ist verbraucht und lässt sich nicht länger aufrechterhalten ${ }^{64}$. Das bleibt nolens volens, abgesehen von der erneut im Raum stehenden Frage nach dem sich unter dem Mönchsnamen Theophilus verbergenden Verfasser der ,Schedula', nicht ohne Folgen für die bisher herrschende Datierung, derzufolge sie in der ersten Hälfte des 12 . Jahrhunderts verfasst worden sein soll ${ }^{65}$.

Hier ist daran zu erinnern, daß in der Forschungsliteratur auch frühere, zum Teil bis in karolingische Zeit zurückreichende Datierungen vorgeschlagen worden sind. Bernhard Bischoff hat sich mit ihnen auseinandergesetzt und ist zu dem Schluß gekommen: „Die handschriftliche Überlieferung führt nicht vor 1100 hinauf "66. Damit tut sich ein gewisser Spielraum auf, denn ob die Zeit um 1125 beziehungsweise später, oder um 1100 beziehungsweise früher, etwa 1090 angenommen wird, ist in dieser Zeit des „Umbruchs“ nicht belanglos ${ }^{67}$. Bernhard Bischoff stellt am Ende seiner Untersuchung fest: „[...] und damit ist der Weg frei, bei der Vergleichung dieses Lehrbuchs mit den Techniken und Formen, die sich in den Denkmälern darbieten, auch das 11. Jahrhundert in seiner ganzen Ausdehnung einzubeziehen. ${ }^{c 68}$ In dieser Perspektive können die Frühdatierungen als Indizien dafür verstanden werden, daß nicht die sakramentale Bestimmung der Kunstfertigkeiten an sich, sondern deren etwa um 1100 in der ,Schedula' erfolgte Verschriftlichung die Neuheit gewesen ist.

Grundlegend für das in den Prologen entworfene Idealbild vom Kunstfertigen ist die monastische Lebenswelt, die Papst Gregor der Große zusammen mit dem

${ }^{64}$ Cf. K. G. Beuckers, Rezension von Stiegemann/Westermann-Angerhausen (eds.), Schatzkunst (cf. nt. 4) in: Kunstform 8 (2007), Nr. 10 [www.arthistoricum.net/kunstform/rezension/ausgabe/ 2007/10/10480/cache.off]: „[...] Wenn das urkundlich genannte Stück, das Roger gefertigt hat, nicht der Dom-Tragaltar ist, dann fällt die gesamte Zuschreibung der Gruppe an diesen Namen und die dafür rekonstruierte Vita sowie auch die Zuordnung der,Schedula' zu dieser Gruppe, die ja über den Namen Rogers erfolgt ist. Damit ist die Kunstgeschichte, die diese Bezüge bisher akzeptiert hat, gefordert."

65 Sowie den mit ihr einhergehenden konjunkturellen Annahmen, wie der Einfluß von Rupert von Deutz, die Auseinandersetzung um den Schmuck des Gotteshauses, den Bernhard von Clairvaux in seiner ,Apologie' verwirft, Abt Suger von Saint Denis hingegen fördert, und der Bezug zur Parallele von artes liberales und artes mechanicae in der Lehrschrift ,Didascalicon' von Hugo von Sankt Viktor.

${ }^{66}$ Bischoff, Untersuchungen (nt. 17), 146-149, hier 146; dazu cf. op. cit., 149: „Es besteht, von der Schrift der ältesten Handschriften und der Textentwicklung der Schedula her gesehen, keine Notwendigkeit, mit der Abfassungszeit weit vor das Einsetzen der Überlieferung hinauszugehen, $[\ldots] . "$

${ }^{67}$ Cf. G. Weilandt, Krise des Königshofes - Krise der Kunst? Zum Einfluss gesellschaftlicher Kräfte auf die künstlerischen Traditionen im späten 11. Jahrhundert, in: Jarnut/Wernhoff, Umbruch (nt. 60), 453-467.

${ }^{68}$ Bischoff, Untersuchungen (nt. 17), 149. 
von ihm eingeführten Pastoralauftrag der Bischöfe zum Pfeiler der christlichen Kirche gemacht hat und die als Kulturträger deren Entwicklung bis ins 12. Jahrhundert bestimmt ${ }^{69}$. In der ,Schedula ${ }^{6}$ kommen innovative Aspekte dazu: Die Vorstellung von der Entwicklung der menschlichen Geschicklichkeit als einem von Gott erhaltenen, genealogisch strukturierten und umsonst weiterzugebenden Erbe mit dem dazugehörenden Erbrecht, die als Dichter und Büßer charakterisierte David-Figur, die so weit ich sehe einmalige Auslegung der sieben Gaben des Heiligen Geistes auf die Tätigkeit des Kunstfertigen, die im Betrachter beim Anblick der Bildwerke ausgelöste affektive Wirkung und schließlich das in der Einleitung der ,Schedula“ mit dem Hinweis auf den Zusammenhang von „nützlicher Handbeschäftigung und dem Bedenken ergötzlicher Neuheiten“ angekündigte und in der Zuordnung der drei Bücher und der drei Prologe gegebene Verhältnis von „Praxis“ und „Theorie“70.

Der eingangs bemerkte Sachverhalt, daß die Prologe in den jüngeren Abschriften der ,Schedula' nur vereinzelt aufgenommen worden sind, spricht dafür, daß das in ihnen präsentierte Bild vom Kunstfertigen nach dem Entstehen der beiden ältesten Abschriften, spätestens aber vom 13. Jahrhundert an als unzeitgemäß und unverstanden denn auch nicht mehr rezipiert worden ist.

${ }^{69}$ Cf. P. Brown, Die Entstehung des christlichen Europa, München 1996.

${ }^{70}$ Cf. G. Beaujouan, Réflexions sur les rapports entre théorie et pratique au moyen âge, in: J. E. Murdoch/E. D. Sylla (eds.), The Cultural Context of Medieval Learning. Proceedings of the First International Colloquium on Philosophy, Science and Theology in the Middle Ages, September 1973 (Synthese Library 76), Dordrecht 1975, 437-484, 440 wird Theophilus erwähnt; M. Gibson, The Artes in the Eleventh Century, in: Arts libéraux et philosophie au moyen âge. Actes du IV congrès international de philosophie médiévale, Montréal 27 août-2 septembre 1967 (Publication de l'Institut d’Études Médiévales de Montréal), Paris-Montréal 1969, 121-126 [wiederabgedruckt in: ead., ,Artes' (nt. 56)]. 